\title{
Beyond Graphene: Stable Elemental Monolayers of Silicene and Germanene
}

\author{
Nathanael J. Roome and J. David Carey*
}

Advanced Technology Institute, University of Surrey, Guildford, GU2 7XH, United Kingdom

\section{Abstract}

Two-dimensional materials are one of the most active areas of nanomaterials research. Here we report the structural stability, electronic and vibrational properties of different monolayer configurations of the group IV elemental materials silicene and germanene. The structure of the stable configuration is calculated and for planar and low $(<1 \AA)$ atomic buckling configurations, analysis of the electronic band structure reveals linear band dispersion giving rise to massless Dirac fermions with a Fermi velocity about two-thirds that of graphene. Monolayer stability is shown to be directly attributed to the phonons present with the instability being driven by the out-of-plane ZA and ZO phonon modes. Long momentum relaxation lengths and high carrier mobilities are predicted for silicene and germanene based devices as carrier relaxation via phonon scattering is found to be inhibited as the electron - optical phonon coupling matrix elements are calculated to be small, being about a factor of 25 times smaller than in graphene. The consequences for phonon scattering, high energy electrical transport and integration of elemental monolayers into electronic devices are further discussed.

Keywords: 2D monolayers, silicene, germanene, graphene, electron-phonon coupling, carrier relaxation 


\section{Introduction}

Two-dimensional layered materials ${ }^{1}$ such as graphene ${ }^{2}$, hexagonal boron nitride ${ }^{3}$ and the transition metal dichalcogenides ${ }^{4}$, offer considerable potential to exploit a range of attractive electronic properties and to take advantage of their inherent 2D structure, either as single or few layers, or as part of a layered heterostructure. ${ }^{1-4}$ Graphene is the most widely studied 2D monolayer (ML) material and many of its properties reflect its distinctive low energy electronic band structure. ${ }^{5}$ Around the Dirac point, where the occupied $\pi$ and unoccupied $\pi^{*}$ states cross, the band dispersion is linear leading to quasiparticles that mimic the characteristics of massless Dirac fermions. This results in carriers which propagate with a Fermi velocity, $v_{F}$, that is about $1 / 300^{\text {th }}$ of the speed of light leading to high room-temperature mobilities. Graphene has already demonstrated these properties serving variously as a calibration for universal constants ${ }^{6}$, as a transparent contact in solar cells ${ }^{7}$ and in high frequency electronics with high cut-off frequencies ${ }^{8}$. While there is sustained research effort in the study of graphene, recent attention has turned to alternative 2D materials. Of particular interest are the other group IV elemental materials, silicene and germanene as predominately $\mathrm{sp}^{2}$ near-planar analogues of $\mathrm{Si}$ and Ge, respectively. These materials possess a (near) honeycomb lattice and a general feature of a $2 \mathrm{D}$ honeycomb lattice of identical atoms is the emergence of linear band dispersion behaviour around the Fermi energy at the K point and the appearance of massless Dirac fermions which can lead to high carrier mobility.

To date silicene has been produced on $\operatorname{Ag}(111)$ substrates $^{9,10}$ and $\operatorname{Ir}(111)$ substrate $^{11}$, on $\operatorname{Ag}(110)$ as nanoribbons ${ }^{12}$ and recently on the conductive ceramic $\mathrm{ZrB}_{2}(0001) / \mathrm{Si}(111)^{13}$. An energy gap between the occupied and unoccupied electron states can be engineered in these monolayers through exposure to hydrogen. For example Restrepo et al. have shown that complete hydrogenation of silicene produces silicane, which has an indirect energy gap of 2.94 $\mathrm{eV}^{14}$ However for a $1.6 \%$ tensile strain, the band gap changes to be direct and there is an 18 fold increase in electron mobility to be in excess of $8,550 \mathrm{~cm}^{2} / \mathrm{Vs}$. A hydrogenated form of 
germanene, germanane, has also recently been exfoliated ${ }^{15}$ and it has been proposed that stable germanene may be able to be grown on GaAs, ${ }^{16}$ though confirmation of the growth of germanene on metal substrates is awaited. Ab initio calculations have also recently investigated the properties of stanene, a monolayer version of $\mathrm{sp}^{2}$ tin, and found that it may be a quantum spin Hall insulator with a direct energy gap of $0.3 \mathrm{eV} .{ }^{17}$ Given silicon's pre-eminence as an electronic material, and the possibility of easier incorporation of silicene in devices compared to graphene, knowledge of the electronic and vibrational properties of its 2D analogue, and related materials, is of both fundamental and applied interest. Previous ab initio studies ${ }^{18}$ have demonstrated that both silicene and germanene can adopt two different buckled geometries, referred to as low buckling (LB, less than $1 \AA$ ) and high buckling (HB). In both cases atoms in different triangular sub-lattices are offset from planarity by a total buckling height, $\Delta$, (Figure 1a). Experimental evidence of buckling has been obtained by scanning tunnelling microscopy measurements $^{9-12}$; the extent of which depends on the substrate used.

Key questions that are therefore to be addressed in the present study include the quantification of the buckling for freestanding MLs, its effect on the electronic band structure and phonon dispersion, and the identification of important phonon modes, including Raman active modes. This knowledge will allow us to identify the factors that affect ML stability and further allow us to calculate the electron-phonon coupling (EPC) matrix elements. The interaction between carriers and phonons is one of the most important in condensed matter physics and it plays an important role in electrical transport, where phonon absorption and optical phonon emission at high electric fields strongly affects mobility and thermal transport. ${ }^{19}$ Carrier relaxation in the presence of an electric field is usually described by assuming non-relativistic electron propagation, where the phonons are treated adiabatically within the Born-Oppenheimer approximation (BOA) as frozen displacements of the electronic ground state. In graphene the inverse of the zone-centre optical phonon frequency which plays an 
a)
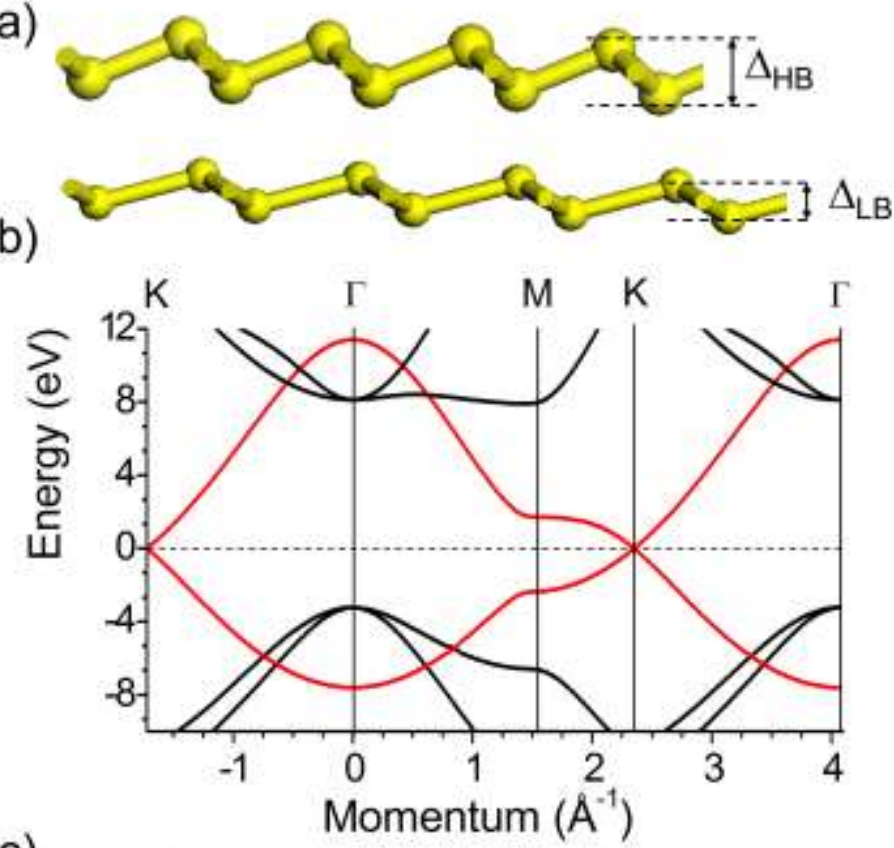

c)
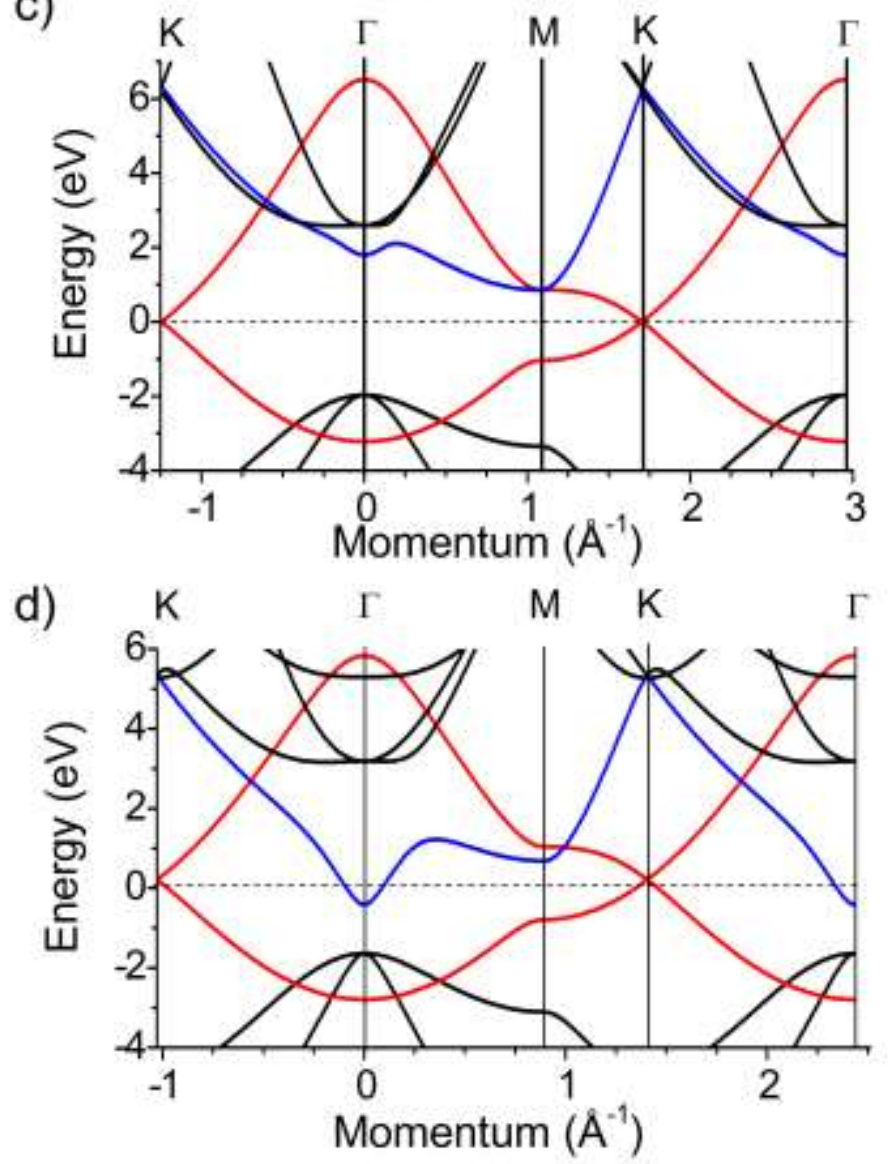

Figure 1. (a) Atomic configuration in non-planar geometry with the atom buckling characterised by total height $\Delta$; each sub-lattice is offset from planarity by a height $\Delta / 2$. HB and LB refer to high and low buckling, respectively. The electronic band structure for free-standing planar monolayers of (b) graphene, (c) silicene and (d) germanene; the occupied $\pi$ and unoccupied $\pi^{*}$ bands are indicated by red lines. The unoccupied 'stray' band, referred to in the text, is indicated by the blue line in (b) and (c); this band appears at much higher energies in graphene. 
Roome and Carey, ACS Appl. Mater. Interfaces 6, 7743 - 7750 (2014)

important role in hot carrier relaxation, is much smaller than the electron momentum relaxation time, $\tau_{R}$, leading to a breakdown of this approximation. ${ }^{20}$ Near the Dirac point in silicene $\tau_{R}$ has been calculated ${ }^{21}$ to be as long as $1.8 \mathrm{ps}$, therefore analysis of the vibrational properties will also determine whether the BOA can be applied to adiabatic carrier relaxation in silicene and germanene. In this study we explore, using ab initio methods, the properties and stability of group IV 2D layered materials. Comparison of silicene and germanene's properties, such as Fermi velocity, band structure and phonon dispersion with graphene will provide new insight into the structural, electronic and vibrational properties of elemental monolayers beyond graphene and their potential insight into their electronic applications.

\section{Computational Details}

The optimised (planar and buckled) atomic arrangements, electronic band structure and phonon dispersion curves are calculated from density functional theory (DFT) and density functional perturbation theory (DFPT) via diagonalization of the dynamical matrix, respectively. A revised Perdew-Burke-Ernzerhof functional ${ }^{22}$ within the generalised gradient approximation using $\mathrm{DMol}^{3}$ (reference 23) and CASTEP $\operatorname{codes}^{24}$ is used. Free-standing MLs are described by their crystallographic space group, $\mathrm{P} 6 / \mathrm{mmm}$ (planar) and $\mathrm{P}-3 \mathrm{~m} 1$ (buckled configurations), with layers separated by a vacuum slab of greater than $10 \AA$ in thickness. Initially the atoms undergo a geometric optimisation by forcing the system into an atomic arrangement which is subsequently allowed to relax into the minimum energy state where the forces on the atoms are less than $0.01 \mathrm{eV} / \AA$. The Brillouin zone undergoes an energy optimisation with a sampling Monkhorst-Pack grid chosen to achieve a k-point separation $<0.025 \AA^{-1}$ (better than $12 \times 12 \times$ 2). Plane wave cut-off energies within CASTEP are set above $400 \mathrm{eV}$. Phonon dispersion curves are calculated using both the finite displacement method ${ }^{24}$ and the linear response

method. ${ }^{25}$ Both methods rely on atomic perturbations of the atom geometry followed by a relaxation to define the vibrational mode. The finite displacement method produces smooth 
Roome and Carey, ACS Appl. Mater. Interfaces 6, 7743 - 7750 (2014)

phonon branches and accurate calculations of vibrational frequencies but can miss kinks or sharp changes. The linear response method captures the finer detail of higher energy vibrational modes but can produce errors across the branches. When calculating the EPC matrix elements it is necessary to examine for kinks in the phonon branches as these are signatures of the presence of Kohn anomalies. Such features are associated with the softening of the vibrational frequencies for phonons that connect states near the Fermi surface; hence a combination of the simulation methods is employed. In hexagonal lattices Kohn anomalies may rise at the zone centre, associated with the double degenerate $\mathrm{E}_{2 \mathrm{~g}}$ phonon mode, or at the zone boundary, associated with the $\mathrm{A}_{1}{ }^{\prime}$ phonon mode. The appearance of negative phonon frequencies in the output of the phonon dispersion calculations is a sign of monolayer instability.

\section{Results and Discussion: Planar Monolayers of Graphene, Silicene and Germanene}

Figure 1b-d shows the electronic band structure for graphene, planar silicene and germanene, where linear band dispersion behaviour around the Dirac point at the $\mathrm{K}$ point in the Brillouin zone, is seen. The $\pi$ and $\pi^{*}$ bands in all three materials (red lines in Figure 1) are associated with the out-of-plane $p_{z}$ molecular orbitals; there are also several $\sigma$ and $\sigma^{*}$ bands associated with in-plane $\mathrm{sp}^{2}$ bonding and anti-bonding orbitals. In Figure 1c $\boldsymbol{\&} \mathbf{d}$, the $\pi$ and $\pi^{*}$ bands in the planar silicene and germanene (red lines) are still observed though the energy separation at the $\Gamma$ point is lower due to the weaker $\pi$ bonding associated with the larger lattice constant. From the slope of the band dispersion around the Dirac point the Fermi velocity is calculated to $8.0 \times 10^{5} \mathrm{~m} / \mathrm{s}$ in graphene, but is lower in planar silicene and germanene, $5.2 \times 10^{5}$ and $5.6 \times 10^{5} \mathrm{~m} / \mathrm{s}$, respectively, (Table 1). The hopping integral, $t$, associated with electron hopping between triangular sub-lattices, is calculated from $v_{F}$ via $t=\frac{2 \hbar v_{F}}{\sqrt{3} a}$, where $a$ is the lattice constant; the values of $t$ are calculated to be $2.50,1.03$ and $1.05 \mathrm{eV}$, respectively. The reduction in $t$ found in silicene and germanene, when compared to graphene, is also associated with 
weaker $\pi$ bonding. The high value of $v_{F}$ and the linear band dispersion leading to massless

Dirac fermions will lead to high carrier mobilities in silicene and germanene (provided that the coupling to phonons is weak, as discussed later).

Table 1. Calculated properties for graphene, silicene and germanene in the planar, low and high buckling forms. The parameters are lattice constant, total atomic buckling $(\Delta)$, energy stability of the atoms relative to bulk crystal, Fermi velocity, highest phonon frequencies at the high symmetry $\Gamma$ and $\mathrm{K}$ points, and electron-phonon coupling matrix elements. The free-standing material stability is predicted based on analysis of these properties.

\begin{tabular}{|c|c|c|c|c|c|c|c|c|}
\hline \multirow{3}{*}{$\begin{array}{l}\text { Atomic } \\
\text { mass }\end{array}$} & & Graphene & \multicolumn{3}{|c|}{ Silicene } & \multicolumn{3}{|c|}{ Germanene } \\
\hline & & 12.01 & & 28.09 & & & 72.64 & \\
\hline & $\begin{array}{l}\text { Zone } \\
\text { point }\end{array}$ & Planar & Planar & $\begin{array}{c}\text { Low } \\
\text { buckling }\end{array}$ & $\begin{array}{c}\text { High } \\
\text { buckling }\end{array}$ & Planar & $\begin{array}{c}\text { Low } \\
\text { buckling }\end{array}$ & $\begin{array}{c}\text { High } \\
\text { buckling }\end{array}$ \\
\hline $\begin{array}{l}\text { Lattice } \\
\text { constant, } \\
(\AA)\end{array}$ & & 2.43 & 3.85 & 3.88 & 2.71 & 4.07 & 4.06 & 2.97 \\
\hline $\begin{array}{l}\text { Buckling } \\
\text { height, } \\
\Delta(\AA)\end{array}$ & & - & - & 0.44 & 2.15 & - & 0.67 & 2.28 \\
\hline $\begin{array}{l}\text { Energy } \\
\text { stability } \\
\text { (eV/atom) }\end{array}$ & & - & 0.62 & 0.45 & 0.61 & 0.54 & 0.28 & 0.38 \\
\hline $\begin{array}{l}\text { Fermi } \\
\text { velocity, } \mathrm{v}_{\mathrm{F}} \\
\left(\times 10^{5} \mathrm{~m} / \mathrm{s}\right)\end{array}$ & & 8.0 & 5.21 & 5.27 & & 5.59 & 5.09 & - \\
\hline $\begin{array}{l}\text { Highest } \\
\text { frequency } \\
\text { vibration, } \\
\omega\left(\mathrm{cm}^{-1}\right)\end{array}$ & $\begin{array}{l}\Gamma \\
\mathrm{K}\end{array}$ & $\begin{array}{l}1566 \\
1377\end{array}$ & $\begin{array}{l}604 \\
475\end{array}$ & $\begin{array}{l}556 \\
503\end{array}$ & $\begin{array}{l}392 \\
216\end{array}$ & $\begin{array}{l}336 \\
307\end{array}$ & $\begin{array}{l}292 \\
274\end{array}$ & $\begin{array}{l}195 \\
106\end{array}$ \\
\hline 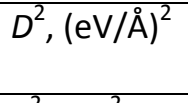 & $\begin{array}{l}\Gamma \\
\mathrm{K}\end{array}$ & $\begin{array}{l}42.6 \\
65.0\end{array}$ & $\begin{array}{l}1.4 \\
2.9\end{array}$ & $\begin{array}{l}1.9 \\
2.7\end{array}$ & - & $\begin{array}{l}0.3 \\
2.4 \\
\end{array}$ & $\begin{array}{l}1.6 \\
1.4\end{array}$ & - \\
\hline$g^{2},\left(\mathrm{eV}^{2}\right)$ & $\begin{array}{l}\Gamma \\
\mathrm{K} \\
\end{array}$ & $\begin{array}{l}0.038 \\
0.076 \\
\end{array}$ & $\begin{array}{l}0.001 \\
0.004 \\
\end{array}$ & $\begin{array}{l}0.002 \\
0.003 \\
\end{array}$ & - & $\begin{array}{l}0.0002 \\
0.002 \\
\end{array}$ & $\begin{array}{l}0.001 \\
0.001 \\
\end{array}$ & - \\
\hline $\begin{array}{l}\text { Predicted } \\
\text { monolayer } \\
\text { stability }\end{array}$ & & Stable & Unstable & Stable & Unstable & Unstable & Stable & Unstable \\
\hline
\end{tabular}

In planar germanene the electronic band structure shows a band (Figure 1d, blue line) crossing the Fermi level around the $\Gamma$ point which results in a finite density of states and quasi-metallic behaviour. There is also an upward shift of $0.35 \mathrm{eV}$ of the Dirac point relative to the Fermi level. The origin of this 'stray' band is the subject of some debate having previously ${ }^{26}$ been assigned to a $\pi^{*}$ anti-bonding band, where it was proposed that in-plane compression pushed this band into the valence band but preserved the cone-like shape of the $\pi$ and $\pi^{*}$ bands. We 
Roome and Carey, ACS Appl. Mater. Interfaces 6, 7743 - 7750 (2014)

believe this assignment to be incorrect, rather we believe that the band is associated with an $\sigma^{*}$ anti-bonding band. Orbital analysis of all the bands crossing the $\Gamma$ point in all three materials has been performed, see Tables S1-S3 in the Supplementary Information, where it shows that the stray band in germanene has $\sigma^{*}$ character. Such analysis consists of identifying a $\sigma$ wavefunction as one where the orbital phase does not change upon a horizontal refection in the plane containing the bond; whereas $\pi$ states are characterised by a change of phase upon $180^{\circ}$ rotation along the direction of the bond. The occupancy of this anti-bonding orbital contributes to the instability of planar germanene. The stability of the planar MLs can be further inferred from analysis of the phonon dispersion curves, Figure 2. In graphene the phonon branches at the $\Gamma$ point are, in order of decreasing frequency, the doubly degenerate longitudinal optical (LO) and transverse optical (TO) modes at $1566 \mathrm{~cm}^{-1}$, the out-of-plane optical (ZO) mode $\left(877 \mathrm{~cm}^{-1}\right)$ and three transverse, longitudinal and out-of-plane acoustic (TA, LA, ZA) modes. At the $\mathrm{K}$ point, the modes present are the TO mode $\left(1337 \mathrm{~cm}^{-1}\right)$, doubly degenerate LO and LA modes $\left(1204 \mathrm{~cm}^{-1}\right)$, TA mode $\left(983 \mathrm{~cm}^{-1}\right)$ and the degenerate ZO and ZA modes $\left(532 \mathrm{~cm}^{-1}\right)$.

Within the $\mathrm{D}_{6 \mathrm{~h}}$ point symmetry of the planar MLs, the $\mathrm{E}_{2 \mathrm{~g}}$ LO mode at the $\Gamma$ point, and the $A_{1}{ }^{\prime}$ TO mode at the $K$ point correspond to the Raman $G$ and defect-activated $D$ peaks, respectively. The corresponding modes in silicene and germanene are given in Table $\mathbf{1}$, and our calculations predict that for free-standing undoped monolayers, respectively, a Raman peak associated with the $E_{2 \mathrm{~g}}$ mode of vibration at around $604 \mathrm{~cm}^{-1}$ (silicene) and $336 \mathrm{~cm}^{-1}$ (germanene), analogous to the $\mathrm{G}$ band along with a defect-activated $\mathrm{D}$ peak at $475 \mathrm{~cm}^{-1}$ (silicene) and $307 \mathrm{~cm}^{-1}$ (germanene). Substantial negative frequencies are observed in the calculated phonon dispersion of silicene and germanene (Figure $\mathbf{2 b}$ and $\mathbf{c}$ ); the exact vibrational mode connected with a particular branch being resolved at the high symmetry $\Gamma$ and K points. For planar silicene negative vibrations are associated with the $\mathrm{ZO}$ mode around the $\Gamma$ point; this out-of-plane vibration causes atomic buckling to increase resulting in the low buckling configuration. 


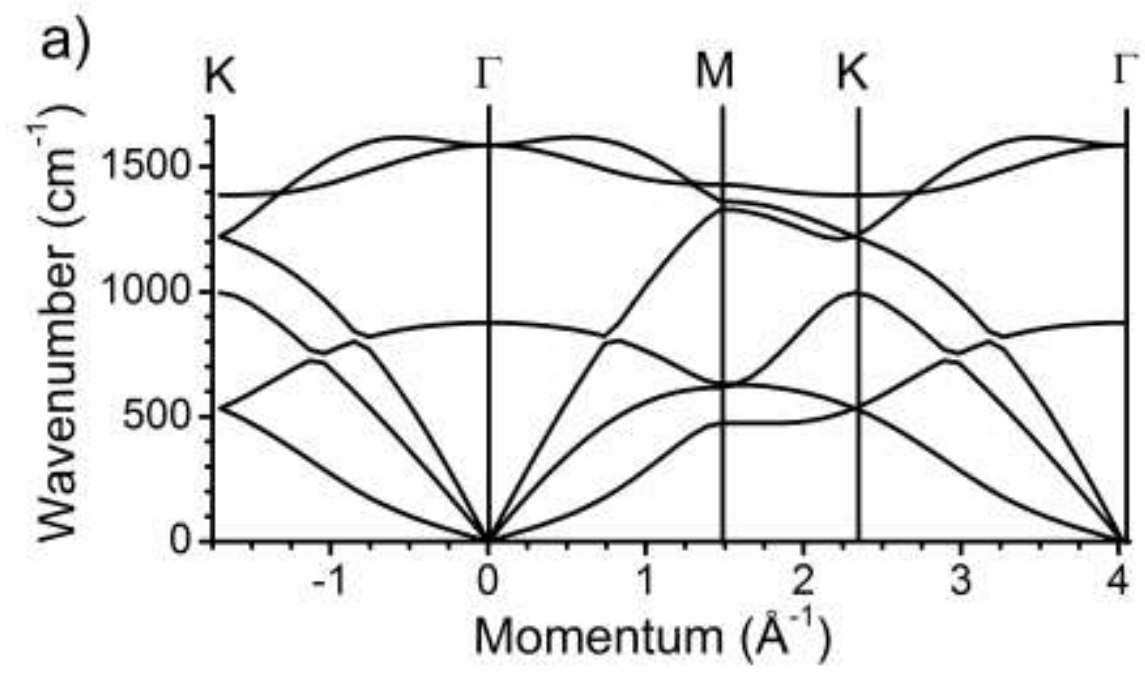

b)
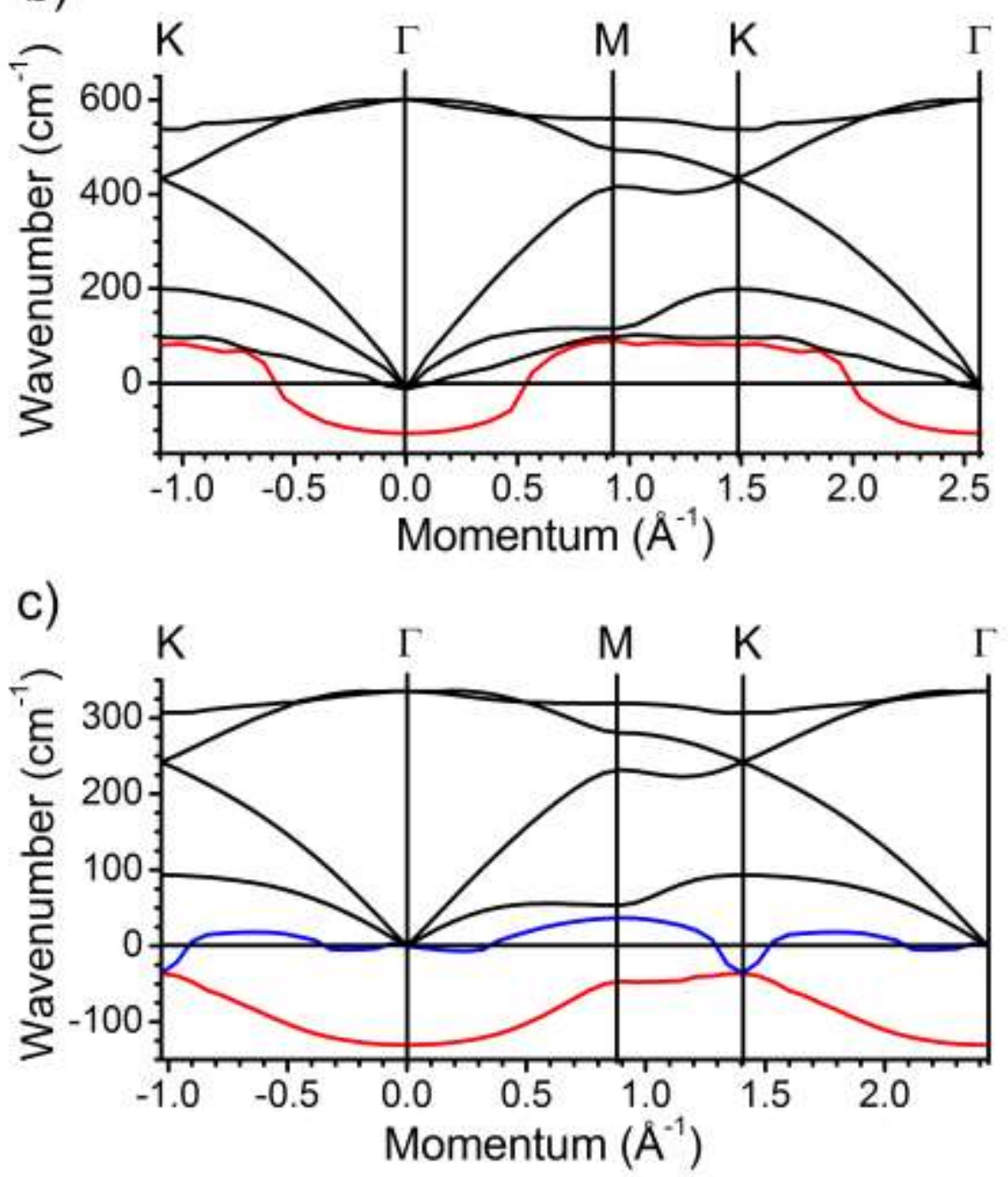

Figure 2. Phonon dispersion curves for (a) graphene, (b) planar silicene and (c) planar germanene monolayers. Negative values of phonon frequencies can be used to infer the stability of the monolayer. The ZO phonon mode is marked in red in (b) and (c) showing the extent of the negative frequencies. The ZA phonon mode in germanene is marked in blue in (c). 
We propose that if the amplitude of this $\mathrm{ZO}$ mode vibration can be suppressed, equivalent to removing the zero point vibrational energy contribution to the overall ML stabilisation energy e.g. by the selection of an appropriate substrate which would dampen the out-of-plane vibrational mode, then planar silicene could be produced. However for germanene the absence of any positive frequencies associated with the $\mathrm{ZO}$ mode, and the negative frequencies with the ZA mode, results in two vibrational modes contributing to ML structural instability. Given the extent of the negative frequencies present associated with the two modes, stable planar freestanding germanene is unlikely.

\section{Results and Discussion: Buckled Monolayers of Silicene and Germanene}

Turning to the buckled configurations, Figure 3, shows the electronic band structures for silicene and germanene in both LB and HB configurations. The minimum energy low bucking configuration in silicene (germanene) is characterised by $\Delta_{\mathrm{LB}}=0.44 \AA(0.67 \AA)$, whereas in the high buckling configuration $\Delta_{\mathrm{HB}}=2.15 \AA(2.28 \AA)$, respectively. Table 1 shows that in terms of relative stability (calculated relative to bulk $\mathrm{Si}$ or $\mathrm{Ge}$ ), the $\mathrm{LB}$ form of silicene is 0.17 eV/atom more stable than the planar form, which itself is comparable in energy to the HB form. In the case of germanene the LB form is again the most stable, being $0.26 \mathrm{eV} /$ atom more stable than the energy of the planar form though in this case the HB form of Ge is much closer in energy to the LB form being only $0.1 \mathrm{eV} /$ atom higher in energy. These stabilisation energies are larger than those calculated by Scalise et al. who calculate using either the local density approximation or the generalised gradient approximation, stabilisation energies of silicene of 14 to 27 meV/atom, respectively. ${ }^{27}$ From Figure 3 in the LB configuration a low energy linear dispersion behaviour is seen around the $K$ point with a Fermi velocity of $5.3 \times 10^{5} \mathrm{~m} / \mathrm{s}$ in silicene and $5.1 \times 10^{5} \mathrm{~m} / \mathrm{s}$ in germanene; similar to the values found in the planar MLs. A $0.6 \times 10^{5} \mathrm{~m} / \mathrm{s}$ decrease in the Fermi velocity from planar to LB germanene is attributed to the increased the distance between the nearest neighbour atoms from $2.351 \AA$ to $2.439 \AA$. 
a)

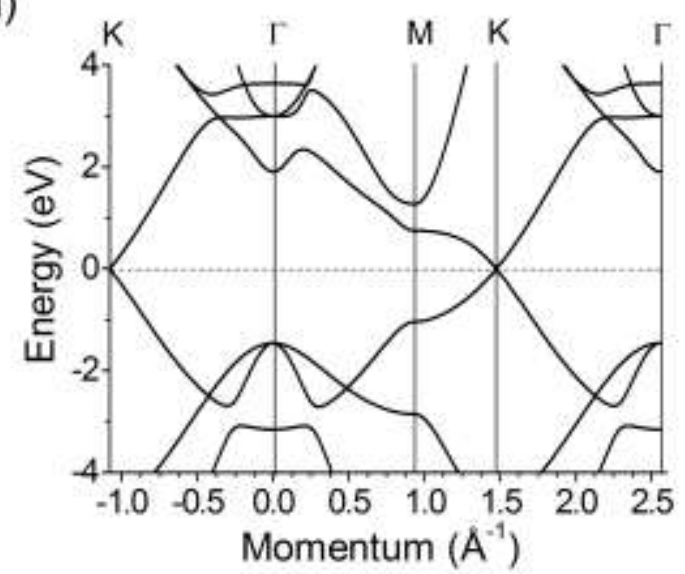

c)

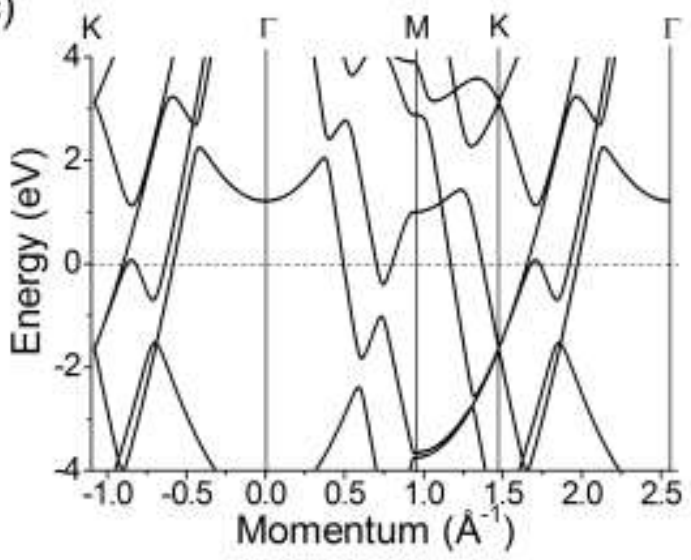

b)

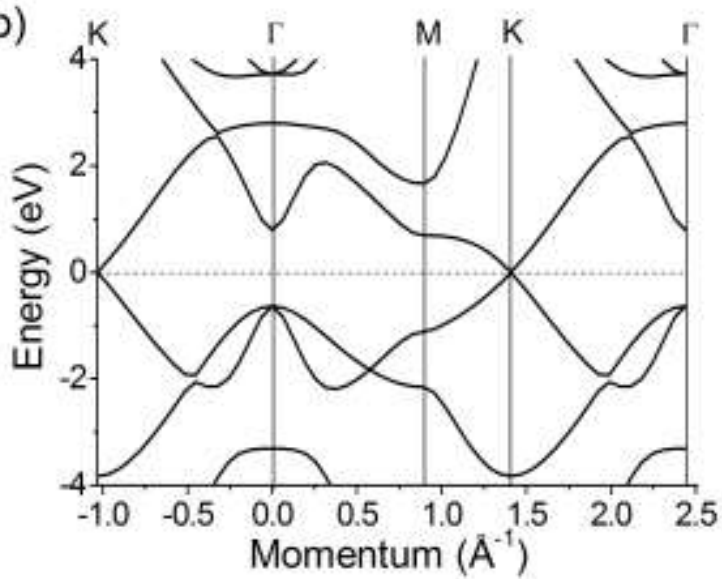

d)

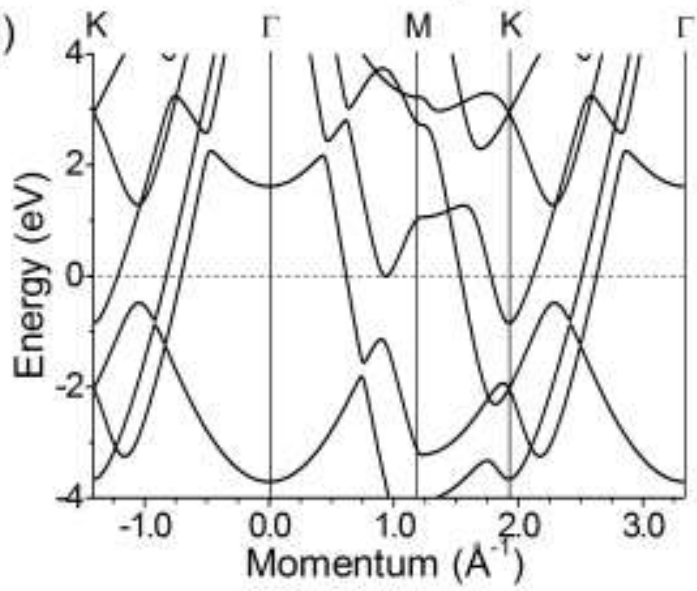

Figure 3. The electronic band structure of (a) LB silicene, (b) LB germanene, (c) HB silicene and (d) HB germanene. Linear dispersion at the $\mathrm{K}$ point is observed in the low buckling configurations but is absent in the high buckling configuration.

Lower values of Fermi velocity were found in the study by Guzmán-Verri and Lew Yan Voon who explored using a first nearest neighbour and second nearest neighbour tight-binding formulism to calculate the band structure. ${ }^{28}$ They found in their planar form of silicene a Fermi velocity of about $10^{5} \mathrm{~m} / \mathrm{s}$ - about half that found in this study - but that in a buckled form the Fermi velocity had reduced to $10^{4} \mathrm{~m} / \mathrm{s}$. In that study the atomic buckling is characterised by half of the Si atoms being vertically displaced from the $\mathrm{Si}(111)$ plane by an amount which is approximately equal to $1.1 \AA$, intermediate between the low- and high buckling found in this $a b$ initio study. In both buckled germanene configurations the stray $\sigma^{*}$ band seen crossing the Fermi level in planar germanene is no longer present. Figure 3 also shows the low energy $\sigma$ and $\sigma^{*}$ bands positions in LB silicene and germanene near the $\Gamma$ point are getting progressively 
Roome and Carey, ACS Appl. Mater. Interfaces 6, 7743 - 7750 (2014)

closer together as a consequence of the buckled geometry. In graphene the $\mathrm{sp}^{2}$ states are constructed from $\mathrm{s}, \mathrm{p}_{\mathrm{x}}$ and $\mathrm{p}_{\mathrm{y}}$ carbon orbitals leading to $\sigma$ state formation; in a fully $\mathrm{sp}^{3}$ material, such as diamond, the states are constructed from a combination of $s, p_{x}, p_{y}$ and $p_{z}$ states. External methods such as thermal annealing or ion implantation ${ }^{29}$ can respectively change the globally or locally $\mathrm{sp}^{3}$ to $\mathrm{sp}^{2}$ fraction in mixed-phased materials. The $\mathrm{p}_{\mathrm{z}}$ orbitals in graphene go onto to form the $\pi$ states the $\pi$ and $\sigma$ states are decoupled from one other. Coupling and admixture to introduce some $\mathrm{sp}^{3}$ character occurs in silicene and germanene as the $\pi$ bonding is weaker, as the bond length is longer, resulting in the loss of planarity. As the atoms in the layer are offset from the atomic plane the usually perpendicular $\mathrm{p}_{z}$ orbital is increasingly interacting with the $\mathrm{sp}^{2}$ orbitals due to the reduced angle between them. This additional interaction contributes to the bonding and results in a mixed-phase material that is predominantly $\mathrm{sp}^{2}$ with a small amount of $\mathrm{sp}^{3}$ character present. The buckling also lowers several point group symmetries $\left(D_{6 h}\right.$ to $\left.D_{3 d}\right)$ and this in turn breaks several energy degeneracies associated with the bands. In the electronic band structure this is characterised by the emergence of anti-crossing bands. The effects are most prominent in germanene as the buckling $(0.67 \AA)$ is $50 \%$ greater than silicene $(0.44 \AA)$.

Previous STM measurements ${ }^{11}$ of silicene grown on $\operatorname{Ir}(111)$ have revealed a $\sqrt{3} \times \sqrt{3}$ low buckled $(0.6 \AA)$ silicene construction on a $\sqrt{7} \times \sqrt{7} \operatorname{Ir}(111)$ superstructure. The periodicity of the silicene layer on $\operatorname{Ir}(111)$ was found to be about $7.2 \AA$, which is larger than $6.6 \AA$; which is $\sqrt{3} \mathrm{x}$ lattice constant for low buckled silicene calculated here. The longer lattice constant, and hence longer $\mathrm{Si}-\mathrm{Si}$ bond, may indicate stabilisation through charge transfer from the Ir substrate via p-d orbital hybridisation. Cinquanta et al. in their STM studies of silicene on $\operatorname{Ag}(111)$ reported a $4 \times 4$ and a $\sqrt{13} \times \sqrt{13}$ superstructure as the two most common atomic arrangements grown at the lower growth temperature of $250^{\circ} \mathrm{C}$; above a growth temperature of $270^{\circ} \mathrm{C}$ a $2 \sqrt{3} \times 2 \sqrt{3}$ arrangement predominates. ${ }^{10}$ The unit cell parameter of the $4 \times 4$ structure 
was measured to be $11.78 \AA$ and incorporated three hexagonal unit cells of silicene with a unit cell parameter of $3.926 \AA$; this value being just $1.2 \%$ larger than that calculated here suggesting the presence of a small amount of tensile strain. However the interaction with the silver substrate is complicated with substrate induced symmetry breaking resulting in $2 / 3$ of the atoms being closer to the surface due to strong $\mathrm{Si}(3 \mathrm{p})$ and $\mathrm{Ag}(5 \mathrm{~d})$ hybridisation. For silicene and germanene in the high buckling form the changes in the band structure make it increasingly difficult to distinguish which bands are crossing one another. In the high buckling configuration the significant movement of the atoms away from planarity allows the spin-orbit interaction to induce energy gaps. This is noticeable at the $\mathrm{K}$ point where a large energy gap appears in both HB silicene and germanene.
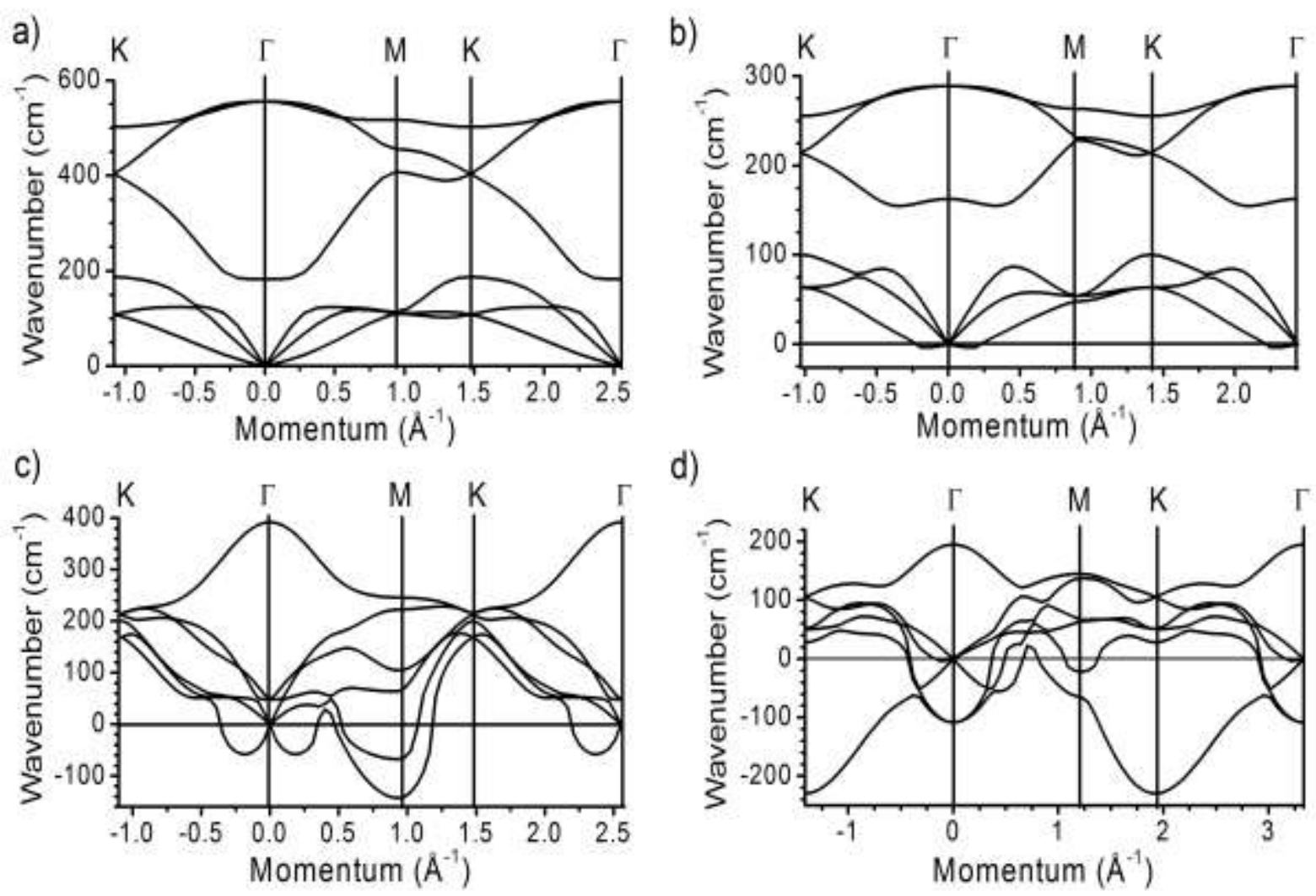

Figure 4. Phonon dispersion curves for (a) LB silicene, (b) LB germanene, (c) HB silicene and (d) HB germanene. The absence of significant negative frequencies for the low buckling cases would suggest they are stable. In the high buckling forms there are substantial LO and TO branches calculated to have negative frequencies, indication that these configurations are unstable. 
Roome and Carey, ACS Appl. Mater. Interfaces 6, 7743 - 7750 (2014)

The phonon dispersion curves in Figure 4 (a) and (b) show that LB silicene and germanene are predicted to be stable with none (in silicene) and only a small extent (germanene) of the phonons having negative frequencies. As summarised in table 1, the frequency of the zone centre phonon in the LB configuration of silicene and germanene is $556 \mathrm{~cm}^{-1}$ and $292 \mathrm{~cm}^{-1}$, respectively. This value is lower than the corresponding mode in the planar monolayer (being 604 and $336 \mathrm{~cm}^{-1}$, respectively); the reduction in the phonon frequency is attributed to the large bond length. Scalise et al. reported similar vibrational modes at $575 \mathrm{~cm}^{-1}$ (silicene) and 285 $\mathrm{cm}^{-1}$ (germanene), though the exact value depends on the choice of exchange-correlation functional employed in their calculations. ${ }^{27}$ Our calculations show that in the low buckling configuration that the defect-activated Raman D peak appears at $503 \mathrm{~cm}^{-1}$ (silicene) and 274 $\mathrm{cm}^{-1}$ (germanene), respectively. The separation in frequency between the $\mathrm{G}$ and $\mathrm{D}$ peaks is far smaller than in graphene and a notable feature of the experimental Raman signal is an asymmetry broadening of the line shape. For example Cinquanta et al. ${ }^{10}$ in their study of silicene containing the $4 \times 4$ and $\sqrt{13} \times \sqrt{13}$ superstructures found an asymmetry line shape at $516 \mathrm{~cm}^{-1}$ accompanied by broad shoulder extending down to about $440 \mathrm{~cm}^{-1}$. The reason for the broadening is a combination of Raman peaks arising from the different atomic configurations. Lu et al. also reported a low frequency Raman mode at $506 \mathrm{~cm}^{-1}$ from a $3.5 \mathrm{~nm}$ thick crystalline nanosheet with $400 \mathrm{~nm}$ diameter. The full-width half-maximum of the Raman band was $30 \mathrm{~cm}^{-1}$ which is larger than that found in bulk crystalline $\mathrm{Si}\left(20 \mathrm{~cm}^{-1}\right) .^{30}$

For the HB configurations (Figure $4 \mathbf{c} \& \mathbf{d}$ ) substantial negative frequencies across much of the Brillouin zone can be seen and this contributes to ML instability. Here there are distinct differences in the order of the phonon modes between planar or LB silicene and germanene. In the HB configuration at the $\Gamma$ point the highest optical branch is now the $\mathrm{ZO}$ mode (at $392 \mathrm{~cm}^{-1}$ in silicene and $195 \mathrm{~cm}^{-1}$ in germanene) and it is predicted to be Raman active. For silicene the TO and LO modes are predicted to have positive frequencies but in germanene they are negative. At the $\mathrm{K}$ point in germanene it is the TO vibrational mode that is calculated to have a 
substantially negative frequency; this implies that it is the in-plane vibrations that are causing high buckling germanene ML to be unstable. These TO and LO vibrations stretch the bond lengths of the highly buckled sheet, moving the atoms into a geometry that would result in germanene breaking-up into islands or nanoribbons.

\section{Electron-Phonon Coupling and Carrier Relaxation in Silicene and Germanene}

Calculation and proper identification of the phonon dispersion has important consequences for the treatment of transport and carrier relaxation. Previously it has been reported ${ }^{20}$ that the use of the Born-Opppenheimer approximation was not valid in the study of graphene as the inverse of the frequency of the zone centre $E_{2 g}$ vibrational mode was very much shorter than the momentum relaxation time of the electrons. Shao et al. have recently calculated $\tau_{\mathrm{R}}$ at $300 \mathrm{~K}$ near the Dirac point for silicene to be $1.8 \mathrm{ps}$ for both electrons and holes ${ }^{21}$; no values are known for germanene. The inverse frequency of the high frequency mode at the $\Gamma$ vibrational mode is calculated here to be $10 \mathrm{fs}$ for LB silicene and $18 \mathrm{fs}$ for LB germanene, so it can be expected that problems with trying to apply the Born-Opppenheimer approximation to understand carrier relaxation in silicene and germanene will also emerge. An alternative carrier relaxation route which does not involve zone centre optical phonons is via coupling to phonons at other high symmetry points. For example, carrier relaxation in graphene can be achieved by coupling of electrons to zone boundary ( $\mathrm{K}$ point) momentum phonons, ${ }^{31}$ hence estimates of electron-phonon coupling strength at the high symmetry points are important. Optical phonon emission occurs when the carrier has exceeded the threshold phonon energy. ${ }^{19}$ For graphene we calculate that this will occur via emission of a zone centre phonon with an energy of $194 \mathrm{meV}$ and a zone boundary phonon of $166 \mathrm{meV}$. For the stable form of silicene the highest phonon energies are calculated to be 69 and $62 \mathrm{meV}$, respectively, and for germanene they are 36-34 meV. As we shall show below an important difference between silicene and germanene when compared to graphene, is the estimation of the efficiency of this alternative relaxation route, 
Roome and Carey, ACS Appl. Mater. Interfaces 6, 7743 - 7750 (2014)

even though the threshold energies for phonon emission in silicene and germanene are lower than in graphene.

Electron-phonon coupling can be defined as the derivative of the hopping integral with respect to the carbon bond distance. The strength of the coupling can be described ${ }^{31,32}$ in terms of the EPC matrix element, $D_{(k+q) n, k m}$, given by

$$
D_{(k+q) n, k m}=\left\langle k+q, n\left|\Delta V_{q}\left[\Delta n_{q}\right]\right| k, m\right\rangle,
$$

where $\Delta \mathrm{V}_{\mathrm{q}}$ and $\Delta \mathrm{n}_{\mathrm{q}}$ are the derivatives of the Kohn-Sham potential and charge density with respect to the displacement along the normal coordinate, respectively, and $|k, n\rangle$ is the electronic Bloch eigenstate at wavenumber $k$ and band $n$. The EPC can also be expressed in terms of the product of $D$ with the phonon characteristic length to give

$$
\left|g_{q}\right|^{2}=\left|D_{q}\right|^{2}\left(\frac{\hbar}{2 M \omega_{q}}\right)
$$

where $M$ is the atomic mass and $\omega_{q}$ is the particular phonon frequency; carrier scattering rates are proportional to $g^{2}$. The units of $D^{2}$ are $\mathrm{eV}^{2} / \AA^{2}$ and those of $g^{2}$ are $\mathrm{eV}^{2}$. Equation (2) simplifies to $g_{q}^{2}=1.402 \times D_{q}^{2} / \omega_{q}$ for graphene, $g_{q}^{2}=0.599 \times D_{q}^{2} / \omega_{q}$ for silicene and $g_{q}^{2}=0.231 \times D_{q}^{2} / \omega_{q}$ for germanene, with $\omega$ measured in $\mathrm{cm}^{-1}$ in all three cases. The EPC matrix elements can be calculated ${ }^{31}$ from the slope of electronic band structure, $\beta$ (which is equal to $\hbar v_{F}$ ) from Figures 1 and 3, and from the slopes of the phonon dispersion curves from Figures 2 and 4 around the high symmetry $\Gamma$ and $K$ points via

$$
D_{q}^{2}=\frac{8 M \omega_{q}}{\sqrt{3} a \hbar} \times \beta \times \frac{d \omega_{q}}{d k}
$$

From Table 1 the value of $D^{2}$ in graphene is calculated to be $42.6 \mathrm{eV}^{2} / \AA^{2}$ for the zone centre $\mathrm{E}_{2 \mathrm{~g}}$ mode and $65.0 \mathrm{eV}^{2} / \AA^{2}$ for the $\mathrm{A}_{1}{ }^{\prime} \mathrm{TO}$ mode at the $\mathrm{K}$ point; these values are in good agreement with previous studies. ${ }^{32}$ For planar silicene and germanene the calculated value of 
Roome and Carey, ACS Appl. Mater. Interfaces 6, 7743 - 7750 (2014)

$D^{2}$ is significantly smaller (1.4 and $0.3 \mathrm{eV}^{2} / \AA^{2}$, respectively). For the LB configurations values of $D^{2}$ of 1.9 and $1.6 \mathrm{eV}^{2} / \AA^{2}$, are calculated for silicene and germanene, respectively. All these values are about a factor of 25 times smaller than those found in graphene. Examining the form of Eq. (3) and comparing the relative contributions of the various terms between planar silicene and graphene, shows that the reduction in the EPC matrix element is primarily associated with the softening of the phonon dispersion curves rather than (i) an increase in atomic mass or lattice constant, (ii) a reduction in phonon frequencies or (iii) decrease in the Fermi velocity. The significantly lower EPC matrix elements in silicene and germanene will result in a lower probability for electrons to relax adiabatically to the ground state through either zone centre or zone boundary phonon emission even though the threshold energies for phonon emission are lower.

\section{Summary}

In conclusion, $a b$ initio calculations of monolayer group IV 2D materials have revealed the presence of linear band dispersion in planar and in low buckling configurations of silicene and germanene with a Fermi velocity that is about two-thirds that found in graphene. Analysis of the phonon dispersion curves shows that the presence of out-of-plane modes in planar silicene and germanene contributes to ML instability. For the high buckled configurations the presence of significant negative phonon frequencies suggest that free-standing monolayers are unlikely to be stable. Estimates of the electron-phonon coupling matrix elements suggest that the interaction of carriers with phonons is reduced by a factor of about 25 when compared to graphene. This will limit adiabatic relaxation of carriers by coupling to zone centre or zone boundary phonons. This will results in long momentum relaxation lengths, high mobilities and will have implications for high field transport studies in group IV monolayer materials. 


\section{ACKNOWLEDGMENT}

N.J.R. would like to acknowledge EPSRC Postgraduate Studentship support. J.D.C. acknowledges previous support from EPSRC.

\section{SUPPORTING INFORMATION}

The supporting information shows the orbital analysis of the band structure of graphene, planar silicene and planar germanene at the $\Gamma$ point in the Brillouin zone. This information is available free of charge via the Internet at http://pubs.acs.org/

*Corresponding Authors. Address correspondence to N.Roome@surrey.ac.uk (N.J.R.) and David.Carey@surrey.ac.uk (J.D.C.)

\section{References}

[1] Geim, A. K.; Grigorieva, I. V.; van der Waals Heterostructures, Nature 2013, 499, 419425 .

[2] Novoselov, K. S.; Geim, A. K.; Morozov, S. V.; Jiang, D.; Zhang, Y.; Dubonos, S. V.; Grigorieva, I. V.; Firsov, A. A. Electric Field Effect in Atomically Thin Carbon Films Science 2004, 306, 666-669.

[3] Dean, C. R.; Young, A. F.; Meric, I.; Lee, C.; Wang, L.; Sorgenfrei, S.; Watanabe, K.; Taniguchi, T.; Kim, P.; Shepard, K. L. et al. Boron Nitride Substrates for High-Quality Graphene Electronics Nat. Nanotechnol. 2010, 5, 722-726.

[4] Mak, K. F.; Lee, C.; Hone, J.; Shan, J.; Heinz, T. F. Atomically Thin $\mathrm{MoS}_{2}$ : A New Direct-gap Semiconductor Phys. Rev. Lett. 2010, 105, 136805.

[5] Novoselov, K. S.; Geim, A. K.; Morozov, S. V.; Jiang, D.; Katsnelson, M. I.; Grigorieva, I. V.; Dubonos, S. V.; Firsov, A. A. Two-dimensional Gas of Massless Dirac Fermions in Graphene Nature 2005, 438, 197-200.

[6] Tzalenchuk, A.; Lara-Avila, S.; Kalaboukhov, A.; Paolillo, S.; Syväjärvi, M.; Yakimova, R.; Kazakova, O.; Janssen, T. J. B. M.; Fal'ko, V. I.; Kubatkin, S. Towards a Quantum Resistance Standard Based on Epitaxial Graphene Nat. Nanotechnol. 2010, 5, 186-189. 
[7] Wang, X.; Zhi, L.; Mullen, K. Transparent Conductive Graphene Electrodes for DyeSensitized Solar Cells Nano Lett. 2008, 8, 323-327.

[8] Lin, Y. M.; Dimitrakopoulos, C; Jenkins, K. A.; Farmer, D. B.; Chiu, H. Y.; Grill, A.; Avouris, P. 100 GHz Transistors from Wafer-scale Epitaxial Graphene, Science 2010, 327,662 .

[9] Vogt, P.; De Padova, P.; Quaresima, C.; Avila, J.; Frantzeskakis, E.; Asensio, M. C.; Resta, A.; Ealet, B.; Le Lay, G. Silicene: Compelling Experimental Evidence for Graphenelike Two-Dimensional Silicon Phys. Rev. Lett. 2012, 108, 155501.

[10] Cinquanta, E.; Scalise, E.; Chiappe, D.; Grazianetti, C.; van den Broek, B.; Houssa, M.; Fanciulli, M.; Molle, A. Getting through the Nature of Silicene: An $\mathrm{sp}^{2}-\mathrm{sp}^{3}$ TwoDimensional Silicon Nanosheet, J. Phys. Chem. C 2013, 117, 16719-16724.

[11] Meng, L.; Wang, Y.; Zhang, L.; Du, S.; Wu, R.; Li, L.; Zhang, Y.; Li, G.; Zhou, H.; Hofer, W. A. et al. Buckled Silicene Formation on $\operatorname{Ir}(111)$ Nano Lett. 2013, 13, 685-690.

[12] De Padova, P.; Kubo, O.; Olivieri, B.; Quaresima, C.; Nakayama, T.; Aono, M.; Le Lay, G. Multilayer Silicene Nanoribbons Nano Lett. 2012, 12, 5500-5503.

[13] Fleurence, A.; Friedlein, R.; Ozaki, T.; Kawai, H.; Wang, Y.; Yamada-Takamura, Y. Experimental Evidence for Epitaxial Silicene on Diboride Thin Films Phys. Rev. Lett. 2012, 108, 245501.

[14 ] Restrepo, O. D.; Mishra, R.; Goldberger, J. E.; Windl, W. Tunable Gaps and Enhanced Mobilities in Strain-Engineered Silicane J. Appl. Phys. 2014, 115, 033711

[15] Bianco, E.; Butler, S.; Jiang, S.; Restrepo, O. D.; Windl, W.; Goldberger, J. E.; Stability and Exfoliation of Germanane: A Germanium Graphane Analogue ACS Nano 2013, 7, 4414-4421.

[16] Kaloni, T. P.; Schwingenschlögl, U. Epitaxial Growth of Germanene on a GaAs (0001) Substrate: A First Principles Approach, J. Appl. Phys. 2013, 114, 184307.

[17] Xu, Y.; Yan, B.; Zhang, H-J.; Wang, J.; Xu, G.; Tang, P.; Duan, W.; Zhang, S-C. LargeGap Quantum Spin Hall Insulators in Tin Films Phys. Rev. Lett. 2013, 111, 136804.

[18] Cahangirov, S.; Topsakal, M.; Akturk, E.; Şahin, H.; Ciraci, S. Two- and Onedimensional Honeycomb Structures of Silicon and Germanium Phys. Rev. Lett. 2009, 102, 236804. 
[19] Dorgan, V.E.; Behnam, A.; Conley, H.J.; Bolotin, K.I.; Pop, E. High-Field Electrical and Thermal Transport in Suspended Graphene Nano Lett. 2013, 13, 4581-4586.

[20] Pisana, S.; Lazzeri, M.; Casiraghi, C.; Novoselov, K. S.; Geim, A. K.; Ferrari, A. C.; Mauri, F. Breakdown of the Adiabatic Born-Oppenheimer Approximation in Graphene Nat. Mater. 2007, 6, 198-201.

[21] Shao, Zhi-Gang; Ye, Xue-Sheng; Yang, Lei; Wang, Cang-Long, First-principles Calculation of Intrinsic Carrier Mobility of Silicene, J. Appl. Phys. 2013, 114, 093712.

[22] Hammer, B.; Hansen, L. B.; Nørskov, J. K. Improved Adsorption Energetics Within Density Functional Theory Using Revised Perdew-Burke-Ernzerhof Functionals Phys. Rev. B 1999, 59, 7413-7421.

[23] Delley, B. From Molecules to Solids with the DMol ${ }^{3}$ Approach, J. Chem. Phys. 2000, $113,7756-7764$.

[24] Segall, M. D.; Lindan, P. J. D.; Probert, M. J.; Pickard, C. J.; Hasnip, P. J.; Clark; S. J.; Payne; M. C. First-principles Simulation: Ideas, Illustrations and the CASTEP code $J$. Phys.: Condens. Matter 2002, 14, 2717-2744.

[25] Baroni, S.; de Gironcoli, S.; Dal Corso, A.; Giannozzi, P. Phonons and Related Properties of Extended Systems from Density Functional Perturbation Theory Rev. Mod. Phys. 2001, 73, 515-562.

[26] Houssa, M.; Pourtois, G.; Afanas'ev, V. V.; Stesmans, A. Electronic Properties of TwoDimensional Hexagonal Germanium Appl. Phys. Lett. 2010, 96, 082111.

[27] Scalise, E.; Houssa, M.; Pourtois, G.; van den Broek, B.; Afanas'ev, V.; Stesmans, A. Vibrational Properties of Silicene and Germanene Nano Research 2013, 6, 19-28.

[28] Guzmán-Verri, G.G.; Lew Yan Voon, L.C. Electronic Structure of Silicon-based Nanostructures Phys. Rev. B 2007, 76, 075131.

[29] Khan, R. U. A.; Carey, J. D.; Silva, S. R. P.; Jones, B. J.; Barklie, R. C. Electron Delocalization in Amorphous Carbon by Ion Implantation Phys. Rev. B 2001, 63, 121201.

[30] Lu, Z; Zhu, J.; Sim, D.; Zhou, W; Shi,W.; Hng, H.H.; Yan, Q Synthesis of Ultrathin Silicon Nanosheets by using Graphene Oxide as Template, Chem. Mater. 2011, 23, 52935295.

[31] Lazzeri, M.; Piscanec, S.; Mauri, F.; Ferrari, A. C.; Robertson, J. Electron Transport and Hot Phonons in Carbon Nanotubes Phys. Rev. Lett. 2005, 95, 236802. 
[32] Piscanec, S.; Lazzeri, M.; Robertson, J.; Ferrari, A. C.; Mauri, F. Optical Phonons in Carbon Nanotubes: Kohn Anomalies, Peierls Distortions and Dynamic Effects Phys. Rev. B 2007, 75, 035427.

\section{Table of Contents Figure}
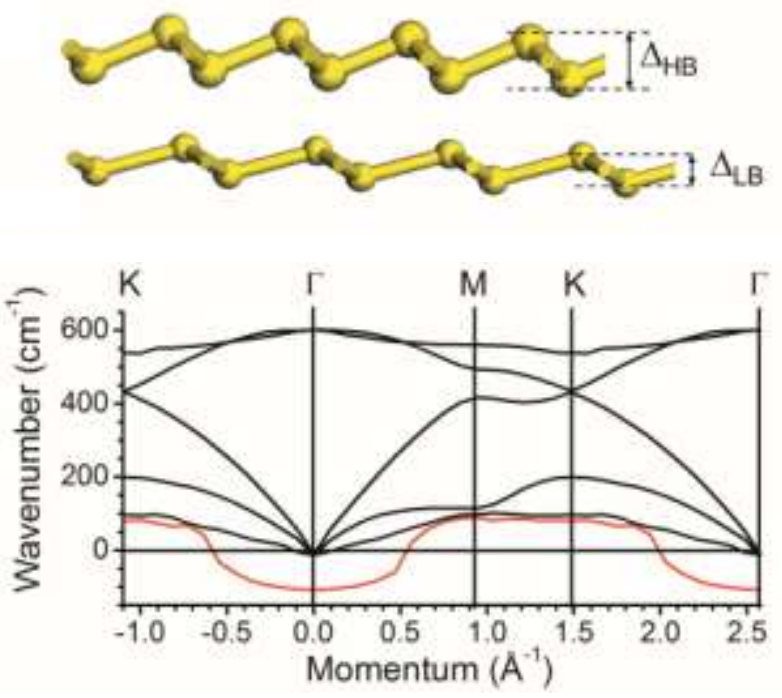
Supporting Information

\section{Beyond Graphene: Stable Elemental Monolayers of Silicene and Germanene}

\section{Nathanael J. Roome and J. David Carey*}

Table S1: Orbital analysis of the band structure in graphene, corresponding to Figure $1 \mathrm{~b}$.

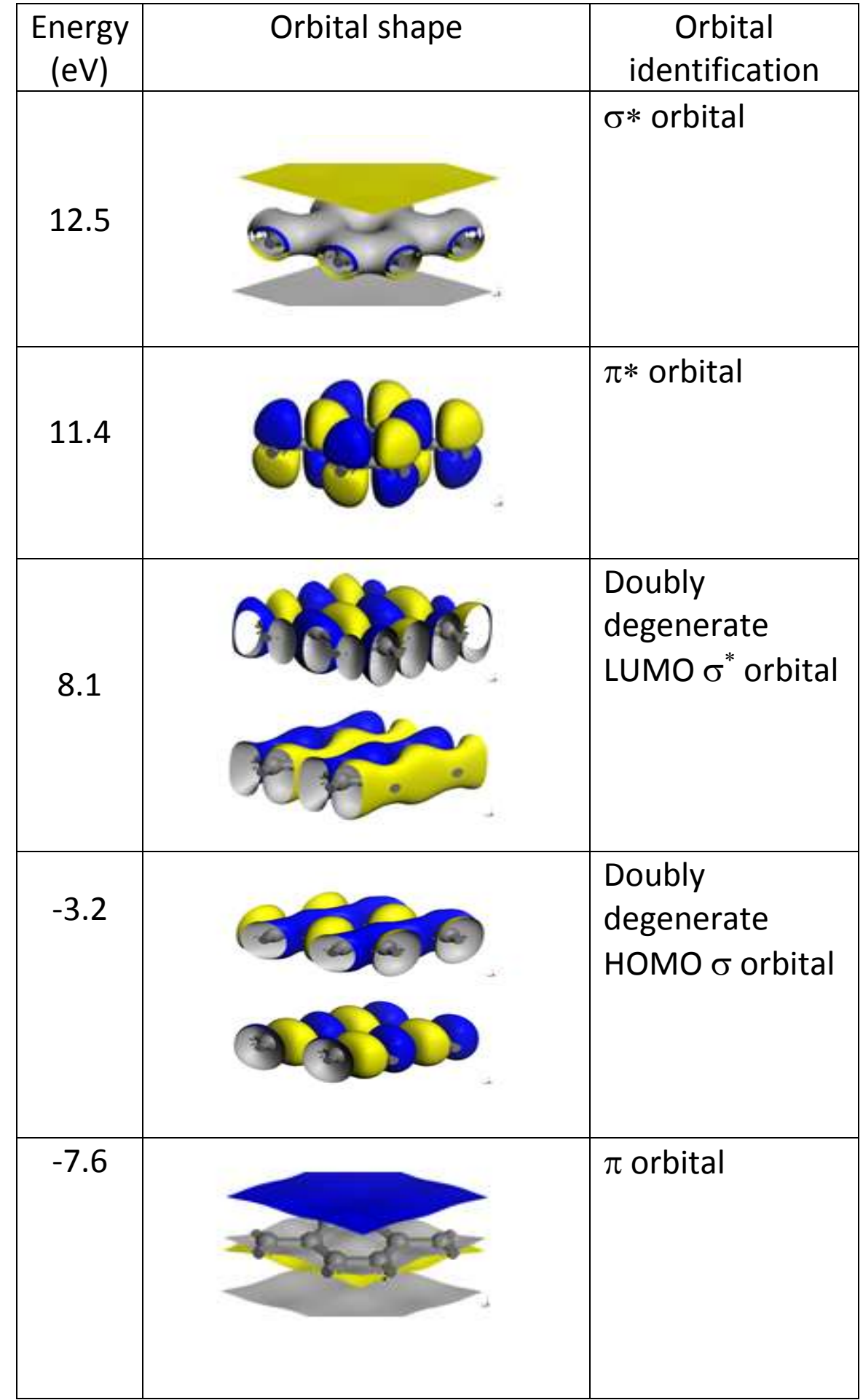


Table S2: Orbital analysis of the band structure in planar silicene, corresponding to Figure 1c.

\begin{tabular}{|c|l|l|}
\hline $\begin{array}{c}\text { Energy } \\
\text { (eV) }\end{array}$ & \multicolumn{1}{c|}{$\begin{array}{c}\text { Orbital } \\
\text { identification }\end{array}$} \\
\hline 6.5 & & \begin{tabular}{l}
$\pi *$ orbital \\
\hline 2.6
\end{tabular} \\
\hline 1.8 &
\end{tabular}


Table S3: Orbital analysis of the band structure in planar germanene, corresponding to Figure $1 \mathrm{~d}$.

\begin{tabular}{|c|c|l|}
\hline $\begin{array}{c}\text { Energy } \\
(\mathrm{eV})\end{array}$ & Orbital shape & \multicolumn{1}{c|}{$\begin{array}{c}\text { Orbital } \\
\text { identification }\end{array}$} \\
\hline 5.2 & & \begin{tabular}{l}
$\pi *$ orbital \\
\hline 3.2
\end{tabular} \\
\hline-0.4 &
\end{tabular}

